\title{
Toxoplasmosis is a Risk Factor for Acquiring SARS-CoV-2 Infection and a Severe Course of Covid-19 Disease in The Czech and Slovak Population: A Preregistered Exploratory Internet Cross-Sectional Study
}

\author{
Jaroslav Flegr ( $\nabla$ flegr@cesnet.cz) \\ Charles University Faculty of Science: Univerzita Karlova Prirodovedecka fakulta https://orcid.org/0000-0002-0822- \\ 0126
}

\section{Research Article}

Keywords: Covid-19, risk factors, SARS-CoV-2, symptoms, zoonosis, pets, cat.

Posted Date: August 19th, 2021

DOI: https://doi.org/10.21203/rs.3.rs-819824/v1

License: (c) (i) This work is licensed under a Creative Commons Attribution 4.0 International License. Read Full License 


\section{Abstract \\ Background}

Latent toxoplasmosis, i.e. a lifelong infection with the protozoan parasite Toxoplasma gondii, affects about a third of human population worldwide. In the past ten years, numerous studies had shown that infected subjects have a significantly higher incidence of mental and physical health problems and are more prone to exhibiting the adverse effects of various diseases.

\section{Methods}

A cross-sectional internet study was performed on a population of 4,499 (786 Toxoplasma-infected) subjects and looked for factors which positively or negatively affect the risk of SARS-CoV-2 infection and likelihood of a severe course of Covid-19.

\section{Results}

Logistic regression and partial Kendall correlation controlled for sex, age, and size of the place of residence showed that latent toxoplasmosis had the strongest effect on the risk of infection $(\mathrm{OR}=1 \cdot 50)$ before sport $(\mathrm{OR}=1 \cdot 30)$, and borreliosis (1.27). It also had the strongest effect on the risk of severe course of infection (Tau $=0 \cdot 146)$, before autoimmunity, immunodeficiency, male sex, keeping a cat, being overweight, borreliosis, higher age, or chronic obstructive pulmonary disease. Toxoplasmosis augmented the adverse effects of other risk factors but was not the proximal cause of the effect of cat keeping on higher likelihood of Covid infection and higher severity of the course of infection because the effect of cat keeping was observed also (and especially) in a subset of Toxoplasma-infected subjects (Tau $=0.153)$. Effects of keeping a cat were detected only in subjects from multi-member families, suggesting that a cat could a vector for the transmission of SARS-CoV-2 within a family.

\section{Conclusions}

Toxoplasmosis is currently not considered a risk factor for Covid-19 and Toxoplasma-infected subjects are neither informed about their higher risks nor prioritised in vaccination programs. Because toxoplasmosis affects a large segment of the human population, its impact on Covid-19-associated effects on public health could be considerable.

\section{Background}

About one-third of world's population is infected by the protozoan parasite Toxoplasma gondii. The definitive host of Toxoplasma are cats (or any felines), but its intermediate host can be any warm-blooded animal, including humans. In immunocompetent human hosts, a short phase of acute toxoplasmosis spontaneously resolves into a latent phase. It is widely believed that during this phase, bradyzoites, the slowly reproducing stage of Toxoplasma, survive in tissue cysts localised mainly in immune-privileged organs and tissues for the duration of host's life, waiting for the host to be eaten by a cat.[1] The bradyzoites in cysts can, however, be reactivated into rapidly dividing tachyzoites in immunocompromised subjects, for example in AIDS patients or persons after transplantation or anticancer treatment.[2] This can result in the formation of brain lesions and, if untreated, lead to fatal toxoplasmic encephalitis.[3] For a long time, latent toxoplasmosis has been considered asymptomatic in immunocompetent subjects, which is why no effort was invested into finding a drug that would kill the bradyzoites in cysts and treat latent toxoplasmosis. This attitude is now slowly changing.[4] For example, ocular toxoplasmosis, an important cause of infectious uveitis, was believed to be associated with the relatively 
rare congenital toxoplasmosis [5] but current data strongly suggests that is a relatively common effect of postnatally acquired toxoplasmosis, the most common form of toxoplasmosis. It is estimated that in Europe and the USA about $2 \%$ of subjects with "latent" toxoplasmosis have scars or active lesions in the retina.[6-8] Even more serious is the situation in South America, where there are more virulent strains of Toxoplasma and the highest diversity of Toxop/asma strains.[9] In this region, about $20 \%$ of Toxoplasma-seropositive subjects probably have ocular toxoplasmosis.[10] Moreover, ocular toxoplasmosis is not the only serious sequel of postnatal Toxoplasma infection. Latent Toxoplasma infection is often also associated with various inflammatory diseases [11, 12], autoimmune diseases [13], and even some types of cancer. $[14,15]$ In the past twenty years, numerous studies had shown that latent toxoplasmosis has important adverse effects on the mental and physical health of infected subjects.[16-18] Over a hundred studies showed that latent toxoplasmosis strongly affects (according to meta-analytic studies nearly triples) the risk of schizophrenia. Later, a similar and possibly even stronger effect of toxoplasmosis was described in relation to other mental health disorders, including the obsessivecompulsive disorder, Attention deficit hyperactivity disorder, and autism.[19, 20]

In the past decade, several studies showed that latent toxoplasmosis affects both mental and physical health. The first evidence was merely indirect: researchers noticed that infected subjects show clear psychological symptoms of chronic stress, which explained most earlier observed differences in the personality profile and behaviour of Toxoplasma-infected versus Toxoplasma-free subjects.[21] These changes, which go in the opposite direction in men and women [22, 23] were at first interpreted as products of the parasite's manipulative activity aimed at enhancing transmission from the intermediate host (usually a mouse) to the definitive host (a cat) by predation.[24] A large cross-sectional study performed on 333 infected subjects and 1,153 controls showed that Toxoplasma-infected subjects reported higher rates of 77 from a list of 134 disorders.[25] Infected subjects also scored significantly worse on 28 out of 29 health-related variables included in the study. Similar results were obtained in an ecological (correlational) study that was based on WHO data on the incidence of disease and disease burden in 88 WHO-member states.[26] The results showed that the Disability Adjusted Life Year (DALY) for 23 out of 128 diseases and disease categories correlated with the prevalence of latent toxoplasmosis in individual countries even after controlling for per capita GDP, latitude, and humidity. In 29 European countries,

differences in the prevalence of toxoplasmosis turned out to be responsible for $23 \%$ of total variability in disease burden in a model containing the three abovementioned covariates. The strongest associations were observed with cardiovascular diseases, perinatal conditions, and congenital abnormalities (which probably reflects the effect of congenital, not latent toxoplasmosis). Nevertheless, a strong positive association was observed also with filariasis, measles, and leukaemia. Such a broad range of observed associations suggests that the effect of latent toxoplasmosis is rather nonspecific. It has also been shown that toxoplasmosis makes individuals prone to various adverse factors, including fatigue, faster aging, and smoking.[27] It is also known that Toxoplasma modifies the functioning of the host's immune system, especially by increasing the concentration of certain lymphokines (most notably IL-10) [28-31] and changing the counts of various immunocytes.[32]

The main aim of the present study was to explore whether latent toxoplasmosis has any effect on the risk of SARS-CoV-2 infection and the course of Covid-19 disease. To this purpose, the probability of being diagnosed with Covid-19, severity of the course of the disease, and the incidence of specific symptoms of the disease were compared between 4,499 participants of a large internet survey.

\section{Methods}

Participants were recruited by a Facebook-based snowball method.[33] Calls for participation in the study were published about 15 times on the Facebook page of Labbunnies, a 23,000-member group of Czech and Slovak nationals willing to take part in evolutionary psychology studies and help with recruiting participants of such studies, as well as on the author's personal Facebook and Twitter accounts. The Qualtrics questionnaire that was used to gather data contained Facebook 'share' and 'like' buttons, so that participants could help recruit other participants by pressing these buttons. In 
total, the buttons were pressed 12,000 times between 17 October 2020 and 3 March 2021. In total, data from 52,000 respondents were obrtained. The invitation as well as the informed consent on the first page of the questionnaire contained only most general information about the aims of the study and contents of the questionnaire. The subjects were informed that the study examines which factors affect the risk and course of Covid-19 infection and what is the opinion of people about anti-epidemic measurements. Participants were also informed that their participation is voluntary, that they can skip any questions they might find uncomfortable and terminate their cooperation at any point simply by closing the web page. Only subjects who consented to participate in the study by pressing the corresponding button were allowed to take the questionnaire. Respondents were not paid for their participation in the study, but after finishing the 20-minute questionnaire, they received information about the results of related studies. The study was anonymous, but participants had the option of providing their e-mail addresses for a future longitudinal study (about $42 \%$ did) or could ask for their data to be erased after completing the questionnaire (about $2 \%$ did). Data collection was performed in accordance with the relevant guidelines and regulations and the project, including the method of obtaining from all participants informed consent with participation in this anonymous study, was approved by the Institutional Review Board of the Faculty of Science, Charles University (Komise pro práci s lidmi a lidským materiálem Prírodovědecké Fakulty Univerzity Karlovy) No. 2020/25. The study was preregistered at The Open Science Framework: https://doi.org/10.17605/OSF.IO/VWXJE

\section{Questionnaire}

The Qualtrics survey consisted of three parts related to three different projects (Risk and protective factors, Opinions of the Czech public on the anti-epidemic measurements, and the effect of priming by studying graphs of Covid victims on opinions regarding anti-epidemic measures). In the present study, only responses to questions related to Covid-19 risks and protective factors were inspected and analysed. Respondents were asked about their sex, age, size of their place of residence (scale 1-5, 0: under 1,000 inhabitants, 1: 1-5,000 inhabitants, 2: 5-50,000 inhabitants, 3: 50-100,000 inhabitants, 4: 100-500,000 inhabitants, 5: over 500,000 inhabitants), and how many persons live with them in the same household. The subjects indicated whether they had already contracted Covid-19 by choosing from five answers (1: 'No', 2: 'Yes, I was diagnosed with it', 3: 'Yes, but I was not diagnosed with it', 4: 'I am awaiting test results', 5: 'No, but I was in a quarantine'). For the purpose of the current study, answers 1 and 5 were coded as 0 (uninfected with Covid), answer 2 as 1 (Covid patients), and answers 3 and 4 were coded as NA (data not available). The respondents were also asked whether they had ever been tested in a laboratory for toxoplasmosis and/or borreliosis and what their results were (1: 'I do not know, I am not sure', 2: 'No, I was tested and the result was negative', 3: 'Yes - I was tested and the result was positive'). For both toxoplasmosis and borreliosis, the questionnaire was pre-set to indicate the first response 'I do not know, I am not sure' as a default. Similarly, respondents were asked about their Rh status (positive/negative/'I do not know, I am not sure') and blood group (A/B/AB/0/'I do not know, I am not sure'). Respondents were also asked to indicate which risks and protective factors apply to them (for a list of the corresponding binary variables, see column 1 of Table 1). Those who had been diagnosed with Covid-19 were also asked to rate the severity of the course of the disease on a five-point scale (1: no symptoms, 2: like a mild flu, 3: like a severe flu, 4: I was hospitalised, 5: I was treated at an ICU). They also had to check which symptoms they experienced during the course of the Covid infection (for a list of the corresponding binary variables, see column 1 of Table 3).

\section{Statistical analyses}

Before embarking on any analyses, all subjects who completed the questionnaire for the second or third time (they were asked about this at the end of the questionnaire for purposes of the planned longitudinal study), those who answered all or nearly all questions by the same code, and those who finished the questionnaire in less than six minutes were filtered out. After this filtration, the set contained 29,345 records. Only the subjects who were negatively or positively tested for toxoplasmosis (4,499 subjects) were included in the present study. 
Statistical analysis was performed with the Statistica v. 10.0. (descriptive statistics, t-tests) and R v. 3.3.1[34] (all other tests) packages. To compute the partial Kendall correlation, the ppcor package [35] and the the package Explorer 1.0 was used.[36] Correction for multiple tests was done using Benjamini-Hochberg procedure with false discovery rate pre-set to 0.10.[37]

\section{Results}

The final set contained 1,085 men, 142 (13.09\%) of whom were infected with Toxoplasma and 131 (12.07\%) diagnosed with Covid, and 3,414 women, 644 (18.86\%) of whom were infected with Toxoplasma and 339 (9.93\%) diagnosed with Covid. The incidence of other potential risk factors is shown in Table 1.

A significant difference in age between men (38.9, s.d. 10.9) and women (40.0, s.d. 10.5) $\left(t_{4497}=-2 \cdot 86, p=0 \cdot 0043\right)$ and between Toxoplasma-free $(39 \cdot 3$, s.d. $10 \cdot 5)$ and Toxoplasma-infected $(42 \cdot 7$, s.d. $10 \cdot 1)$ women $\left(t_{3412}=-7 \cdot 41, p<0 \cdot 0001\right)$, but not between Toxoplasma-free $(38 \cdot 7$, s.d. $10 \cdot 7)$ and Toxoplasma-infected $(40 \cdot 0$, s.d. $11 \cdot 8)$ men $\left(t_{1083}=-1 \cdot 25, p=0 \cdot 211\right)$ existed in the population under study. No association between age and Covid in either men or women $(p>0 \cdot 38)$ was detected. 
Table 1

Incidence of risk and protective factors for Covid-19 in men and women

\begin{tabular}{|c|c|c|c|c|c|c|c|c|c|c|c|}
\hline & \multicolumn{4}{|c|}{ Women } & \multicolumn{7}{|l|}{ Men } \\
\hline & \multicolumn{2}{|c|}{ no Covid } & \multicolumn{2}{|c|}{ Covid } & \multicolumn{2}{|c|}{ no Covid } & \multicolumn{2}{|c|}{ Covid } & \multirow[t]{2}{*}{ OR } & \multirow[t]{2}{*}{ C.I. 95} & \multirow[t]{2}{*}{$\mathrm{p}$} \\
\hline & $\mathrm{N}$ & $\%$ & $\mathrm{~N}$ & $\%$ & $\mathrm{~N}$ & $\%$ & $\mathrm{~N}$ & $\%$ & & & \\
\hline Toxoplasmosis & 563 & $18 \cdot 31$ & 81 & $23 \cdot 89$ & 115 & $12 \cdot 05$ & 27 & $20 \cdot 61$ & $1 \cdot 50$ & $\begin{array}{l}1 \cdot 19- \\
1.89\end{array}$ & 0.0007 \\
\hline Borreliosis & 410 & $19 \cdot 85$ & 57 & $24 \cdot 26$ & 108 & $14 \cdot 57$ & 17 & $17 \cdot 00$ & $1 \cdot 27$ & $\begin{array}{l}0.96- \\
1.68\end{array}$ & 0.0881 \\
\hline Rh-positivity & 1953 & $76 \cdot 41$ & 219 & $76 \cdot 31$ & 490 & $75 \cdot 85$ & 61 & $74 \cdot 39$ & 0.98 & $\begin{array}{l}0 \cdot 76- \\
1 \cdot 26\end{array}$ & 0.8854 \\
\hline A blood group & 989 & $37 \cdot 26$ & 123 & $41 \cdot 14$ & 236 & 34.05 & 33 & $35 \cdot 87$ & $1 \cdot 16$ & $\begin{array}{l}0.93- \\
1 \cdot 44\end{array}$ & $0 \cdot 1795$ \\
\hline B blood group & 568 & $21 \cdot 40$ & 50 & $16 \cdot 72$ & 156 & $22 \cdot 51$ & 22 & 23.91 & $0 \cdot 82$ & $\begin{array}{l}0 \cdot 62- \\
1.07\end{array}$ & $0 \cdot 1368$ \\
\hline AB blood group & 247 & $9 \cdot 31$ & 34 & $11 \cdot 37$ & 91 & $13 \cdot 13$ & 13 & $14 \cdot 13$ & $1 \cdot 19$ & $\begin{array}{l}0 \cdot 86- \\
1 \cdot 65\end{array}$ & 0.2929 \\
\hline 0 blood group & 850 & $32 \cdot 03$ & 92 & $30 \cdot 77$ & 210 & $30 \cdot 30$ & 24 & $26 \cdot 09$ & 0.91 & $\begin{array}{l}0 \cdot 73- \\
1 \cdot 15\end{array}$ & 0.4396 \\
\hline Being overweight & 739 & $35 \cdot 11$ & 90 & $31 \cdot 03$ & 258 & $42 \cdot 64$ & 35 & 33.02 & $0 \cdot 74$ & $\begin{array}{l}0.57- \\
0.95\end{array}$ & 0.0169 \\
\hline Diabetes & 75 & $3 \cdot 56$ & 7 & $2 \cdot 41$ & 28 & $4 \cdot 63$ & 8 & $7 \cdot 55$ & 0.96 & $\begin{array}{l}0.52- \\
1.76\end{array}$ & 0.8921 \\
\hline $\begin{array}{l}\text { Cardiovascular } \\
\text { problems }\end{array}$ & 131 & $6 \cdot 22$ & 16 & $5 \cdot 52$ & 65 & $10 \cdot 74$ & 9 & $8 \cdot 49$ & $0 \cdot 81$ & $\begin{array}{l}0 \cdot 50- \\
1 \cdot 30\end{array}$ & 0.3820 \\
\hline Asthma & 1846 & $87 \cdot 70$ & 256 & $88 \cdot 28$ & 544 & 89.92 & 89 & 83.96 & $1 \cdot 13$ & $\begin{array}{l}0 \cdot 80- \\
1.59\end{array}$ & 0.4908 \\
\hline $\begin{array}{l}\text { Chronic obstructive } \\
\text { pulmonary disease }\end{array}$ & 49 & $2 \cdot 33$ & 5 & $1 \cdot 72$ & 16 & $2 \cdot 64$ & $1 \cdot 89$ & $0 \cdot 67$ & $\begin{array}{l}0 \cdot 27- \\
1 \cdot 63\end{array}$ & 0.3737 & \\
\hline Immunosuppression & 289 & $13 \cdot 73$ & 40 & $13 \cdot 79$ & 52 & $8 \cdot 60$ & 9 & $8 \cdot 49$ & $1 \cdot 00$ & $\begin{array}{l}0 \cdot 70- \\
1 \cdot 42\end{array}$ & 0.9929 \\
\hline Allergy & 548 & $26 \cdot 03$ & 77 & $26 \cdot 55$ & 150 & $24 \cdot 79$ & 28 & $26 \cdot 42$ & $1 \cdot 06$ & $\begin{array}{l}0 \cdot 81- \\
1 \cdot 37\end{array}$ & 0.6798 \\
\hline Autoimmunity & 168 & $14 \cdot 35$ & 25 & $11 \cdot 57$ & 26 & $8 \cdot 72$ & 6 & $9 \cdot 09$ & $0 \cdot 75$ & $\begin{array}{l}0 \cdot 45- \\
1 \cdot 24\end{array}$ & 0.2545 \\
\hline Living alone & 285 & $9 \cdot 30$ & 28 & $8 \cdot 28$ & 160 & $16 \cdot 91$ & 23 & $17 \cdot 56$ & 0.96 & $\begin{array}{l}0 \cdot 70- \\
1 \cdot 32\end{array}$ & 0.8027 \\
\hline Tobacco smoking & 415 & $19 \cdot 71$ & 54 & $18 \cdot 62$ & 148 & $24 \cdot 46$ & 23 & $21 \cdot 70$ & 0.89 & $\begin{array}{l}0 \cdot 66- \\
1 \cdot 19\end{array}$ & 0.4341 \\
\hline $\begin{array}{l}\text { Marihuana } \\
\text { consumption }\end{array}$ & 56 & $2 \cdot 66$ & 6 & $2 \cdot 07$ & 40 & $6 \cdot 61$ & 14 & $13 \cdot 21$ & $1 \cdot 49$ & $\begin{array}{l}0 \cdot 88- \\
2.53\end{array}$ & $0 \cdot 1367$ \\
\hline $\begin{array}{l}\text { Daily alcohol } \\
\text { consumption }\end{array}$ & 126 & 5.99 & 16 & $5 \cdot 52$ & 97 & $16 \cdot 03$ & 14 & $13 \cdot 21$ & $0 \cdot 86$ & $\begin{array}{l}0 \cdot 55- \\
1 \cdot 33\end{array}$ & 0.4887 \\
\hline
\end{tabular}




\begin{tabular}{|c|c|c|c|c|c|c|c|c|c|c|c|}
\hline & Wome & & & & Men & & & & & & \\
\hline Frequent singing & 269 & $12 \cdot 78$ & 39 & $13 \cdot 45$ & 50 & $8 \cdot 26$ & 14 & $13 \cdot 21$ & $1 \cdot 21$ & $\begin{array}{l}0 \cdot 87- \\
1 \cdot 70\end{array}$ & $0 \cdot 2612$ \\
\hline Sport & 620 & $29 \cdot 45$ & 92 & $31 \cdot 72$ & 274 & $45 \cdot 29$ & 60 & $56 \cdot 60$ & $1 \cdot 30$ & $\begin{array}{l}1 \cdot 03- \\
1.65\end{array}$ & 0.0300 \\
\hline $\begin{array}{l}\text { Cold water } \\
\text { swimming }\end{array}$ & 205 & $9 \cdot 74$ & 37 & $12 \cdot 76$ & 102 & $16 \cdot 86$ & 19 & $17 \cdot 92$ & $1 \cdot 32$ & $\begin{array}{l}0.95- \\
1 \cdot 84\end{array}$ & 0.0989 \\
\hline Frequent sauna use & 94 & 8.03 & 20 & $9 \cdot 26$ & 37 & $12 \cdot 42$ & 8 & $12 \cdot 12$ & $1 \cdot 16$ & $\begin{array}{l}0 \cdot 70- \\
1.93\end{array}$ & 0.5558 \\
\hline $\begin{array}{l}\text { Vitamins and } \\
\text { supplements }\end{array}$ & 753 & $35 \cdot 77$ & 133 & $45 \cdot 86$ & 289 & $47 \cdot 77$ & 60 & $56 \cdot 60$ & 0.62 & $\begin{array}{l}0.49- \\
0.78\end{array}$ & 0.0001 \\
\hline Wearing glasses & 1101 & $60 \cdot 36$ & 169 & $61 \cdot 90$ & 371 & $67 \cdot 82$ & 67 & $66 \cdot 34$ & 0.96 & $\begin{array}{l}0 \cdot 75- \\
1 \cdot 24\end{array}$ & 0.7723 \\
\hline Dog keeping & 365 & $44 \cdot 19$ & 78 & $49 \cdot 37$ & 93 & $51 \cdot 10$ & 21 & $45 \cdot 65$ & 0.82 & $\begin{array}{l}0 \cdot 56- \\
1 \cdot 18\end{array}$ & 0.282 \\
\hline Cat keeping & 381 & $46 \cdot 13$ & 82 & $51 \cdot 90$ & 82 & $45 \cdot 05$ & 17 & $36 \cdot 96$ & $1 \cdot 16$ & $\begin{array}{l}0 \cdot 80- \\
1.68\end{array}$ & 0.4303 \\
\hline
\end{tabular}

The last four columns show the results of logistic regression with Covid-19 infection as a dependent variable, age, sex, size of the place of residence as covariates, and one of risk/protective factors listed in the first column as the independent variable. Results shown in bold were significant after correction for multiple tests.

The risk of acquiring toxoplasmosis depends on certain confounding factors, such as sex, age, and size of the place of residence. Therefore, the association between potential risk factors and protective factors was analysed using a logistic regression with binary variable Covid as a dependent variable, sex, age, size of place of residence as covariates, and one of the analysed factors as the independent variable. The results are shown in the last three columns of Table 1. Toxoplasmosis, with an odds ratio of 1.50 (C.I.95 $=1 \cdot 19-1 \cdot 89, \mathrm{p}=0.0007$, risk ratio $1.40\left(\mathrm{Cl}_{95}: 1 \cdot 05-1 \cdot 80\right)$ for women and $1.91\left(\mathrm{Cl}_{95}: 1 \cdot 20-3.05\right)$ for men, turned out to be the most serious risk factor for being diagnosed with Covid.

It is known that Rh-negative subjects are more prone to experiencing the negative effects of toxoplasmosis than Rhpositive subjects are.[38-40] Therefore, also a more complex model containing not only sex, age, size of the place of residence, and toxoplasmosis, but also the $\mathrm{Rh}$ factor and $\mathrm{Rh}$-toxoplasmosis interaction was analysed. Logistic regression showed a significant effect of toxoplasmosis $\left(\mathrm{OR}=1 \cdot 45, \mathrm{Cl}_{95}: 1 \cdot 06-2 \cdot 10, \mathrm{p}=0 \cdot 046\right)$, but not $\mathrm{Rh}(\mathrm{p}=0 \cdot 86)$ or $\mathrm{Rh}-$ toxoplasmosis interaction $(p=0 \cdot 84)$.

Respondents who had been diagnosed with Covid-19 were asked to rate the course of their disease and indicate which symptoms they experienced during their illness. Figure 1 shows that Toxoplasma-infected subjects of both sexes had a more serious course of Covid. Partial Kendall correlation (Table 2) showed that toxoplasmosis represents a more pronounced risk factor for a severe course of Covid-19 than compromised autoimmunity, immunodeficiency, male sex, cat keeping, being overweight, borreliosis, higher age, or chronic obstructive pulmonary disease does.

One could speculate that keeping a cat could be just a proxy for being Toxoplasma-infected and indeed, the prevalence of toxoplasmosis in subjects who do keep a cat $(24.6 \%)$ was markedly higher than in those who do not $(15 \cdot 5 \%)\left(\mathrm{Chi}^{2}=15 \cdot 5\right.$, $p<0.0001)$. To test this hypothesis, the partial Kendall correlation tests was performed separately for Toxoplasma-free and Toxoplasma-infected subjects (Table 2, the last four columns). Existence of the cat-keeping effects (i.e. increased likelihood of contracting Covid-19 and of having a severe course of the disease in the cat-keepers) in the Toxoplasma- 
infected subpopulation suggests that keeping a cat is a real risk factor for Covid-19, not just a side-effect of a higher probability of having toxoplasmosis. In fact, the strength of the cat-keeping effect was twice higher in the Toxoplasmainfected $(\mathrm{Tau}=0.153)$ than in Toxoplasma-free subjects $(\mathrm{Tau}=0.084)$. One could speculate that a cat might be able to transmit Covid among family members. Members of one family could moreover be infected repeatedly and the virus could adapt to the similar genotype of (genetically related) members of the same family; both of the above scenarios could then result in a more severe course of the disease. Indeed, separate analyses for 194 respondents who lived in multi-member families showed that keeping a cat increases the risk of a severe course of Covid-19 (Tau =0·116, $p=0 \cdot 017)$. The same analysis for 22 respondents who lived alone showed no such increase; in fact, it showed a nonsignificant effect in the opposite direction (Tau $=-0.028, p=0.867)$.

Analogical analyses of the risk of acquiring SARS-CoV-2 infection showed no significant effect of cat keeping neither in people who live in multi-member families nor in those who live alone. However, even here a positive non-significant effect of cat keeping in 3,983 respondents living in multi-member families $\left(\mathrm{OR}=1 \cdot 210, \mathrm{Cl}_{95}: 0.82-1 \cdot 78, \mathrm{p}=0.332\right)$ and a negative non-significant effect in 496 respondents living alone $\left(O R=0.692, \mathrm{Cl}_{95}: 0 \cdot 20-2 \cdot 46, \mathrm{p}=0.568\right)$ were found.

The results also showed that many factors taken into consideration in this study had a much stronger effect on the risk of severe course of Covid in Toxoplasma-infected subjects than in those who were Toxoplasma-free: borreliosis (Tau $=0.212$ vs. 0.010 ), being overweight (Tau $=0 \cdot 140$ vs. 0.089), cardiovascular diseases (Tau =0.170 vs. 0.049), asthma (Tau =0.133 vs. 0.039), chronic obstructive pulmonary disease (Tau $=0.219$ vs. 0.013$)$, allergy (Tau $=0.089$ vs. 0.012), compromised autoimmunity (Tau $=0.254$ vs. 0.074 ), and cat keeping (Tau $=0.153$ vs. 0.0840 ). Conspicuous exceptions were male sex (Tau $=0.095$ vs. 0.112 ) and immunodeficiency (Tau $=0.067$ vs. 0.160$)$. The protective effect of sport was also stronger in the Toxoplasma-free than in the Toxoplasma-infected subset (Tau $=-1 \cdot 16 \mathrm{vs} .0 \cdot 028)$. 
Table 2

Correlation between various risk/protective factors and severity of Covid-19 disease

\begin{tabular}{|c|c|c|c|c|c|c|c|c|c|c|}
\hline & \multicolumn{2}{|l|}{ All } & \multicolumn{2}{|l|}{ Women } & \multicolumn{2}{|l|}{ Men } & \multicolumn{2}{|c|}{ Toxo-free } & \multicolumn{2}{|c|}{ Toxo-infected } \\
\hline & Tau & $p$ & Tau & $\mathrm{p}$ & Tau & $\mathrm{p}$ & Tau & $p$ & Tau & $\mathrm{p}$ \\
\hline Sex & $0 \cdot 116$ & 0.0002 & NA & NA & NA & NA & $0 \cdot 112$ & 0.0017 & 0.095 & $0 \cdot 1713$ \\
\hline Age & 0.093 & 0.0032 & $0 \cdot 124$ & 0.0010 & 0.025 & 0.6763 & 0.066 & 0.0654 & $0 \cdot 137$ & 0.0494 \\
\hline $\begin{array}{l}\text { Size of place of } \\
\text { residence }\end{array}$ & 0.009 & $0 \cdot 7815$ & $-0 \cdot 002$ & 0.9575 & 0.039 & 0.5093 & 0.000 & 0.9895 & 0.065 & 0.3477 \\
\hline Toxoplasmosis & $0 \cdot 146$ & 0.0000 & $0 \cdot 150$ & 0.0001 & 0.123 & 0.0376 & NA & NA & NA & NA \\
\hline Borreliosis & 0.094 & 0.0123 & $0 \cdot 120$ & 0.0088 & 0.030 & 0.6529 & $0 \cdot 010$ & $0 \cdot 8146$ & $0 \cdot 212$ & 0.0301 \\
\hline Rh-positivity & 0.036 & $0 \cdot 3097$ & 0.035 & $0 \cdot 3929$ & 0.064 & 0.3950 & 0.022 & 0.5941 & 0.098 & 0.2112 \\
\hline A blood group & 0.019 & 0.5892 & 0.000 & 0.9945 & 0.079 & 0.2715 & 0.032 & 0.4210 & -0.052 & 0.4932 \\
\hline B blood group & -0.008 & $0 \cdot 8101$ & $-0 \cdot 010$ & $0 \cdot 7940$ & 0.009 & $0 \cdot 8991$ & 0.012 & 0.7680 & $-0 \cdot 069$ & 0.3606 \\
\hline AB blood group & 0.021 & 0.5371 & 0.093 & 0.0210 & $-0 \cdot 160$ & 0.0254 & $-0 \cdot 002$ & 0.9586 & 0.080 & 0.2909 \\
\hline 0 blood group & -0.028 & $0 \cdot 4278$ & $-0 \cdot 050$ & $0 \cdot 2142$ & 0.031 & 0.6694 & -0.043 & $0 \cdot 2834$ & 0.048 & 0.5260 \\
\hline Being overweight & $0 \cdot 104$ & 0.0012 & $0 \cdot 107$ & 0.0052 & $0 \cdot 116$ & 0.0558 & 0.089 & 0.0147 & $0 \cdot 140$ & 0.0505 \\
\hline Diabetes & 0.004 & $0 \cdot 8922$ & $-0 \cdot 021$ & 0.5777 & 0.038 & 0.5301 & 0.019 & 0.5929 & $-0 \cdot 022$ & 0.7602 \\
\hline $\begin{array}{l}\text { Cardiovascular } \\
\text { problems }\end{array}$ & 0.063 & 0.0514 & 0.063 & 0.0985 & 0.075 & 0.2194 & 0.049 & $0 \cdot 1762$ & $0 \cdot 170$ & 0.0174 \\
\hline Asthma & 0.052 & $0 \cdot 1055$ & 0.036 & $0 \cdot 3496$ & 0.094 & $0 \cdot 1233$ & 0.039 & $0 \cdot 2815$ & $0 \cdot 133$ & 0.0630 \\
\hline $\begin{array}{l}\text { Chronic } \\
\text { obstructive } \\
\text { pulmonary } \\
\text { disease }\end{array}$ & 0.072 & 0.0252 & 0.073 & 0.0583 & 0.080 & 0.1853 & $0 \cdot 013$ & $0 \cdot 7220$ & $0 \cdot 219$ & 0.0022 \\
\hline Immunodeficiency & $0 \cdot 135$ & 0.0000 & 0.141 & 0.0002 & $0 \cdot 116$ & 0.0558 & $0 \cdot 160$ & 0.0000 & 0.067 & 0.3472 \\
\hline Allergy & -0.015 & 0.6453 & $-0 \cdot 011$ & $0 \cdot 7829$ & -0.025 & 0.6809 & $-0 \cdot 028$ & 0.4376 & 0.025 & 0.7235 \\
\hline Autoimmunity & $0 \cdot 137$ & 0.0004 & $0 \cdot 146$ & 0.0011 & $0 \cdot 112$ & 0.1590 & 0.074 & 0.0874 & 0.254 & 0.0048 \\
\hline Living alone & 0.034 & 0.2794 & 0.092 & 0.0151 & -0.061 & $0 \cdot 2999$ & 0.030 & 0.3960 & 0.073 & $0 \cdot 2990$ \\
\hline Tobacco smoking & 0.061 & 0.0576 & 0.045 & $0 \cdot 2454$ & 0.090 & $0 \cdot 1364$ & $0 \cdot 082$ & 0.0239 & 0.006 & 0.9351 \\
\hline $\begin{array}{l}\text { Marihuana } \\
\text { consumption }\end{array}$ & -0.001 & 0.9777 & -0.082 & 0.0330 & 0.081 & $0 \cdot 1800$ & 0.057 & $0 \cdot 1207$ & $-0 \cdot 195$ & 0.0065 \\
\hline $\begin{array}{l}\text { Daily alcohol } \\
\text { consumption }\end{array}$ & -0.039 & 0.2311 & -0.094 & 0.0138 & 0.067 & 0.2677 & -0.055 & $0 \cdot 1279$ & 0.035 & 0.6289 \\
\hline Frequent singing & $-0 \cdot 032$ & 0.3162 & $-0 \cdot 060$ & 0.1153 & 0.056 & 0.3553 & -0.053 & 0.1459 & $-0 \cdot 001$ & 0.9861 \\
\hline Sport & -0.092 & 0.0044 & $-0 \cdot 112$ & 0.0036 & -0.056 & $0 \cdot 3543$ & $-0 \cdot 116$ & 0.0015 & $-0 \cdot 028$ & 0.6944 \\
\hline $\begin{array}{l}\text { Cold water } \\
\text { swimming }\end{array}$ & -0.047 & $0 \cdot 1447$ & $-0 \cdot 041$ & $0 \cdot 2851$ & -0.055 & 0.3614 & $-0 \cdot 062$ & 0.0883 & $-0 \cdot 041$ & 0.5691 \\
\hline $\begin{array}{l}\text { Frequent use of } \\
\text { sauna }\end{array}$ & -0.036 & $0 \cdot 3550$ & $-0 \cdot 040$ & $0 \cdot 3731$ & -0.037 & 0.6432 & -0.009 & $0 \cdot 8280$ & $-0 \cdot 177$ & 0.0491 \\
\hline
\end{tabular}




\begin{tabular}{|lllllllllll|}
\hline & All & & \multicolumn{2}{c}{ Women } & \multicolumn{2}{c}{ Men } & \multicolumn{3}{c|}{ Toxo-free } & \multicolumn{2}{c|}{ Toxo-infected } \\
\hline $\begin{array}{l}\text { Vitamins and } \\
\text { supplements }\end{array}$ & -0.047 & 0.1425 & -0.029 & 0.4437 & -0.087 & 0.1499 & -0.068 & 0.0628 & 0.034 & 0.6329 \\
\hline Wearing glasses & -0.033 & 0.3244 & 0.005 & 0.9037 & -0.131 & 0.0346 & -0.003 & 0.9346 & -0.069 & 0.3559 \\
\hline $\begin{array}{l}\text { Current dog } \\
\text { keeping }\end{array}$ & 0.004 & 0.9321 & 0.015 & 0.7737 & -0.042 & 0.6694 & 0.049 & 0.3497 & -0.120 & 0.2637 \\
$\begin{array}{l}\text { Current cat } \\
\text { keeping }\end{array}$ & $\mathbf{0 . 1 0 7}$ & $\mathbf{0 . 0 2 0 3}$ & $\mathbf{0 . 1 2 3}$ & $\mathbf{0 . 0 1 9 4}$ & 0.058 & 0.5549 & 0.084 & 0.1052 & 0.153 & 0.1544 \\
\hline
\end{tabular}

The table shows effect size and significance, i.e. partial Kendall Taus and p values. The effect of sex was controlled for age, and size of place of residence, the effect of age for sex, and size of place of residence, and size of place of residence for sex and age; all other effects were controlled for all three covariates. Positive Tau means a more severe course of Covid in subjects reporting the factor listed in the first column. The results in bold were significant after correction for multiple tests. In less numerous men, all factors lost their significance after correction for multiple tests but observed Tau values show that the effects in men are mostly stronger than in women.

Logistic regression controlled for age, sex, and size of the place of residence showed that Toxoplasma-infected participants reported having more symptoms during Covid, but only the presence of chest pain or pressure $(O R=1.96)$ and higher sexual appetence $(\mathrm{OR}=2 \cdot 68)$ were statistically significant after correction for multiple tests (Table 3$)$. On the other hand, even the effects that did not reach the formal level of statistical significance tended to show a higher frequency of symptoms in Toxoplasma-infected than in Toxoplasma-free subjects and were nearly always stronger in men than in women. 
Table 3

Symptoms of Covid-19 in Toxoplasma-infected and Toxoplasma-free subjects

\begin{tabular}{|c|c|c|c|c|c|c|c|c|c|c|c|}
\hline & All & & Wome & & Men & & & & & & \\
\hline & Toxo- & Toxo+ & Toxo- & Toxo+ & Toxo- & Toxo+ & $\mathrm{OR}$ & C.I.95 & $\mathrm{p}$ & $\mathrm{OR}_{\mathrm{W}}$ & $\mathrm{OR}_{\mathrm{M}}$ \\
\hline $\begin{array}{l}\text { Fever }>38^{\circ} \mathrm{C} \\
\left(100 \cdot 4^{\circ} \mathrm{F}\right)\end{array}$ & 0.38 & 0.39 & 0.34 & 0.37 & $0 \cdot 47$ & 0.45 & $1 \cdot 07$ & $\begin{array}{l}0.67- \\
1.7\end{array}$ & 0.789 & 1.09 & 0.99 \\
\hline Fatigue & $0 \cdot 74$ & 0.81 & 0.79 & 0.90 & 0.57 & 0.57 & $1 \cdot 19$ & $\begin{array}{l}0 \cdot 45- \\
3 \cdot 14\end{array}$ & 0.729 & $1 \cdot 30$ & $1 \cdot 22$ \\
\hline Dry cough & 0.42 & 0.53 & 0.44 & 0.50 & 0.38 & 0.64 & $1 \cdot 40$ & $\begin{array}{l}0.89- \\
2 \cdot 2\end{array}$ & $0 \cdot 141$ & $1 \cdot 16$ & 2.97 \\
\hline Shortness of breath & 0.32 & 0.39 & 0.33 & 0.41 & 0.31 & 0.32 & $1 \cdot 19$ & $\begin{array}{l}0.74- \\
1.91\end{array}$ & $0 \cdot 466$ & $1 \cdot 30$ & $1 \cdot 04$ \\
\hline Sore throat & 0.36 & 0.36 & 0.37 & 0.40 & 0.34 & 0.23 & 1.00 & $\begin{array}{l}0.63- \\
1.61\end{array}$ & 0.985 & $1 \cdot 08$ & $1 \cdot 36$ \\
\hline Headache & $0 \cdot 70$ & 0.78 & 0.74 & 0.84 & 0.60 & 0.59 & $1 \cdot 37$ & $\begin{array}{l}0.82- \\
2 \cdot 29\end{array}$ & $0 \cdot 225$ & $1 \cdot 71$ & 0.90 \\
\hline $\begin{array}{l}\text { Chest pain or } \\
\text { pressure }\end{array}$ & 0.32 & 0.51 & 0.35 & 0.53 & $0 \cdot 24$ & 0.45 & 1.96 & $\begin{array}{l}1.24- \\
3 \cdot 13\end{array}$ & 0.004 & $1 \cdot 79$ & $2 \cdot 73$ \\
\hline Other pains & 0.31 & 0.38 & 0.33 & 0.41 & 0.26 & 0.27 & $1 \cdot 20$ & $\begin{array}{l}0.74- \\
1.93\end{array}$ & 0.453 & $1 \cdot 16$ & $1 \cdot 18$ \\
\hline Diarrhoea & 0.28 & 0.36 & 0.29 & 0.36 & 0.25 & 0.36 & $1 \cdot 40$ & $\begin{array}{l}0.86- \\
2 \cdot 26\end{array}$ & $0 \cdot 171$ & $1 \cdot 17$ & $2 \cdot 26$ \\
\hline Conjunctivitis & 0.08 & $0 \cdot 12$ & 0.09 & $0 \cdot 14$ & 0.05 & 0.05 & $1 \cdot 28$ & $\begin{array}{l}0 \cdot 6- \\
2 \cdot 72\end{array}$ & 0.522 & $1 \cdot 22$ & $1 \cdot 84$ \\
\hline Loss of smell & 0.71 & 0.72 & 0.73 & 0.74 & 0.66 & 0.64 & 0.99 & $\begin{array}{l}0.61- \\
1.59\end{array}$ & 0.954 & 0.99 & 0.94 \\
\hline Loss of taste & 0.58 & 0.55 & 0.62 & 0.59 & 0.49 & 0.45 & 0.88 & $\begin{array}{l}0.56- \\
1.39\end{array}$ & 0.593 & 0.89 & 0.87 \\
\hline Middle ear pain & $0 \cdot 12$ & 0.22 & $0 \cdot 14$ & 0.25 & 0.06 & $0 \cdot 14$ & 1.95 & $\begin{array}{l}0.68- \\
5.56\end{array}$ & $0 \cdot 209$ & $1 \cdot 67$ & $5 \cdot 11$ \\
\hline $\begin{array}{l}\text { Problems speaking } \\
\text { and walking }\end{array}$ & 0.03 & 0.05 & 0.03 & 0.06 & 0.04 & 0.05 & $1 \cdot 72$ & $\begin{array}{l}0.6- \\
4.98\end{array}$ & 0.313 & $1 \cdot 32$ & $2 \cdot 18$ \\
\hline Skin rash & 0.08 & $0 \cdot 10$ & $0 \cdot 10$ & 0.09 & 0.02 & $0 \cdot 14$ & $1 \cdot 31$ & $\begin{array}{l}0.6- \\
2 \cdot 87\end{array}$ & $0 \cdot 492$ & 0.77 & $6 \cdot 00$ \\
\hline $\begin{array}{l}\text { Changes in } \\
\text { pigmentation }\end{array}$ & 0.03 & 0.05 & 0.04 & 0.06 & 0.01 & 0.05 & $1 \cdot 81$ & $\begin{array}{l}0.64- \\
5.09\end{array}$ & 0.262 & $1 \cdot 29$ & $4 \cdot 05$ \\
\hline $\begin{array}{l}\text { Higher sexual } \\
\text { appetence }\end{array}$ & 0.03 & 0.08 & 0.03 & 0.06 & 0.04 & $0 \cdot 14$ & $3 \cdot 40$ & $\begin{array}{l}1.42- \\
8 \cdot 17\end{array}$ & 0.006 & $2 \cdot 53$ & $4 \cdot 50$ \\
\hline Other symptoms & 0.27 & 0.25 & 0.29 & 0.27 & $0 \cdot 22$ & $0 \cdot 18$ & 0.93 & $\begin{array}{l}0.56- \\
1.55\end{array}$ & 0.778 & 0.90 & 0.99 \\
\hline
\end{tabular}

Columns 2-7 show reported incidence of particular symptoms (in percents); the last five columns show results logistic regression with a particular symptom as the dependent variable, age, sex, size of place of residence as covariates, and 
toxoplasmosis as the independent variable. The last two columns show OR computed separately for women and men. The results in bold were significant after correction for multiple tests.

\section{Discussion}

Toxoplasma-infected subjects had a higher probability of being diagnosed with Covid-19 and of having a more severe course of the disease; they were more likely to end up hospitalised and more frequently needed to be treated at intensive care units. They more frequently expressed the specific Covid symptoms, especially chest pain and pressing. Surprisingly, Toxoplasma-infected subjects also more frequently reported higher sexual appetence $(p=0.006)$ during Covid-19; this indicator of the faster life strategy of subjects with impaired health [41] might deserve special attention in future studies.

The effects of toxoplasmosis on the risk of SARS-CoV-2 infection and risk of severe course of Covid-19 were the strongest effects detected in the present study. It must be, however, remembered that subjects with various known risk factors, such as being overweight, are probably more careful and actively try to avoid possible sources of the infection. It is important to note that most factors known to be linked to a severe course of Covid decreased (albeit usually non-significantly) the risk of becoming infected. Toxoplasma infection is not, however, considered a factor that could increase the risk of severe course of Covid and Toxoplasma-infected persons therefore do not modify their behaviour so as to minimise the risk of becoming infected with Covid. Consumption of vitamins and food supplements seems to have the strongest protective effect on the risk of becoming infected with Covid, but people who take vitamins probably also apply other measures to avoid the infection, which is why nobody can tell whether vitamins and supplements alone have such a strong protective effect. Sport had the strongest protective effect against a severe course of Covid-19, but it is also a strong risk factor for acquiring the infection, probably because sport activities increase the number of contacts with potential sources of SARSCoV-2 infection.

Our data showed that latent toxoplasmosis was the strongest risk factor for a severe course of Covid-19. It was stronger than the effect of being overweight, cardiovascular disease, or diabetes. The relative strength of the particular effects should be, however, interpreted with caution. It is possible that subjects with the most severe course of Covid (and logically also those who died) did not participate in our questionnaire study. This preselection could probably explain the relatively weak effect of chronic obstructive pulmonary disease.

There has been much speculation and some indications to the effect that pets could be a vector of Covid-19.[42-44] Empirical data shows that cats and dogs can and do acquire symptomatic or asymptomatic SARS-CoV-2 infection.[45, 46] For example, a study performed in Minnesota, USA [47] showed that the seroprevalence in 239 pet cats and 510 pet dogs was $8 \%$ and $1 \%$, respectively, while a German study showed that seroprevalence in cats during the first wave of Covid was $0.65 \%$ and increased to $1.36 \%$ after the second wave.[48] The prevalence in cats was higher in families of Covid-19 patients [49] and there is even direct evidence of human-to-cat transmission within a family.[50,51] On the other hand, it seems that transmission of the virus between pets is rare if any. Similarly, there is no direct evidence for a transmission of SARS-CoV-2 from cats or dogs to humans.[52] It has been suggested that pets might transmit the virus at least within a family, either from infected animals themselves or by touching, e.g., stroking, healthy animals with virus-contaminated fur or paws.[53] Our data seem to support this possibility. Only nonsignificant positive effect of cat keeping was present in respondents from multimember families, and negative, though again nonsignificant, effect of cat keeping was observed in respondents who live alone. However, keeping a cat was found to increases the risk of a severe course of Covid: it was the fifth strongest factor after toxoplasmosis, diagnosed immunodeficiency, autoimmunity, and male sex. The existence of this effect in a subpopulation of Toxoplasma-infected subjects invalidates the hypothesis that cat keeping is only a proxy of Toxoplasma infection (and possibly a better proxy than the presence of anti-lgG antibodies).

Analyses performed separately on the Toxoplasma-free and Toxoplasma-infected subjects had also shown that most factors have a much stronger effect (or exist only) in the Toxoplasma-infected subpopulation. For example, the effect of

Page $12 / 18$ 
borreliosis is $21 \cdot 5$, chronic obstructive pulmonary disease $16 \cdot 8$, cardiovascular diseases $3 \cdot 5$, compromised autoimmunity 3.4, and age 2.1-times stronger in the Toxoplasma-infected than in Toxoplasma-free subjects. This enhancing effect of toxoplasmosis applies also to some protective factors: visits to sauna and marihuana consumption protect Toxoplasmainfected but not Toxoplasma-free subjects from infection. In contrast, the protective effect of sport was about four times stronger in Toxoplasma-free than in Toxoplasma-infected subjects. The most conspicuous amplifying effect of toxoplasmosis was that on borreliosis-Covid interaction. The effect of being diagnosed with borreliosis was the third strongest effect observed in Toxoplasma-infected participants (Tau $=0.212)$, while in Toxoplasma-free subjects, no such effect was observed (Tau $=0 \cdot 010)$. The same phenomenon has previously been observed for interaction between toxoplasmosis, borreliosis, and depression.[54] A cross-sectional study showed that borreliosis has a relatively strong effect on reported depression but was observed only in Toxoplasma-infected participants. Generally, latent toxoplasmosis seems to make human hosts more prone to a wide spectrum of adverse influences, including genetic factors and pathogens.

One can only speculate about the mechanism by which toxoplasmosis influences the risk of SARS-CoV-2 infection and severity of the course of Covid disease, but it is likely that immunomodulation and immunosuppression associated with Toxoplasma infection probably play an important role in it. [28-31] Toxoplasmosis has a strong effect on the concentration of various cytokines, especially IL-10, IL-5, IL-6, and TGF-b. Most studies investigating this issue were performed on laboratory animals and might reflect changes associated with acute or post-acute toxoplasmosis rather than its latent stage, but some studies suggest the existence of specific changes in immunity in humans. It has been shown that many parameters of people with latent toxoplasmosis differ from those of Toxoplasma-free subjects[32]; for example, seropositive women have increased levels of IL-5 and IL-6, and especially (fivefold) IL-12 in comparison to seronegative controls.[55] In immunology outpatients, Toxoplasma-infected women had increased, and men decreased, counts of leukocytes, NK-cells, and monocytes in comparison to corresponding Toxoplasma-free controls.[32] A crosssectional study performed on Czech population had shown that Toxoplasma-infected subjects more frequently report immune disorders, especially immunodeficiency.[25]

\section{Limitations And Strengths Of The Study}

The main limitation of the present study is that the participants were self-selected and probably do not represent a typical Czech population. Social networks, namely Facebook and Twitter, were used to promote the study, which is why it is likely that only subjects who have internet and use these social networks took part in the study. Moreover, many were recruited via the Facebook site of the main investigator, which narrows down the profile of likely participants even further to people interested in biological sciences, evolutionary psychology, and quite possibly also in keeping cats as pets. On the other hand, they also invited their Facebook friends to participate in the study by pressing the share or like buttons at the end of the questionnaire. Our previous results had shown that the composition of participants of similar internet studies was highly similar, if not identical, to representative internet population with respect to the prevalence of 24 neuropsychiatric disorders[56] or religiosity.[57] It is important to emphasise that the informed consent form, as well as the text used for recruitment of participants, mentioned only 'factors that might influence the risk of infection and the course of Covid disease', that is, it made no reference to toxoplasmosis.

Toxoplasmosis status was self-reported by respondents, which may be viewed as a drawback. On the other hand, it has previously been demonstrated that information on toxoplasmosis status provided by 3,827 participants of another internet study nearly perfectly (99.5\%) corresponded to information in our files on subjects tested for toxoplasmosis in our lab.[58] Still, about $60 \%$ of male and $70 \%$ of female respondents recruited via the Facebook-based snowball method were tested for toxoplasmosis elsewhere, mostly in relation to their health problems (49.4\% of men) or pregnancy (37.6\% of women). [59] It is possible that some subjects misreported their Toxoplasma status. Similarly, some respondents who were Toxoplasma-negative during their serological test may have acquired the infection in the meantime. It must be

Page $13 / 18$ 
emphasised, however, that presence of misdiagnosed subjects in the population can result in Type 2, not a Type 1 error, that is, it can increase the risk of failure to detect existing effects but not the risk of detecting non-existing effects.

The strength of the study was the large number of participants and the fact that it was preregistered before the start of data collection. Technically, it was thus designed as a cross-sectional study. On the other hand, Covid-19 is a new disease and infected Czech and Slovak participants probably acquired the infection in autumn 2020, i.e., after being diagnosed with toxoplasmosis. The study thus in fact had the nature of a prospective case-control study and can say at least something about the causal relation between toxoplasmosis and Covid.

\section{Conclusions}

This is an exploratory, albeit preregistered and large, study performed on a rather specific subpopulation. All results must therefore be considered merely preliminary and they must be confirmed by future independent confirmatory studies. The main result of the present study that deserves a special attention is the identification of a new risk factor for SARS-CoV-2 infection and severe course of Covid-19. The factor, latent toxoplasmosis, seems to have a stronger effect than most other factors known to affect the risks of Covid-19. Moreover, it seems to enhance the negative effects of some other adverse factors, some of which had not been suspected of having any impact on the course of Covid, such as borreliosis and keeping a cat. The effect of toxoplasmosis is probably rather nonspecific, akin to what has been observed for other diseases and disorders. It is probably related to the observed changes in the immune system of Toxoplasma hosts. It can only be speculated whether the effects, e.g. the highly increased level of immunosuppressive cytokine IL-10, are just a sideeffect of a latent lifelong infection or part of Toxoplasma's biological adaptations aimed at surviving the attacks of host's immune system. Latent toxoplasmosis affects about one-third of population in both the developed and developing world. The adverse effects of this zoonosis on public health are therefore probably not negligible.

\section{Declarations}

Ethics approval and consent to participate: The study was approved by the Institutional Review Board of the Faculty of Science, Charles University (Komise pro práci s lidmi a lidským materiálem Př́rodovědecké Fakulty Univerzity Karlovy) No. 2020/25. Data collection was performed in accordance with the relevant guidelines and regulations and the project, and all participants read and approved informed consent with participation in this study.

Consent for publication: Not applicable.

Availability of data and materials: The dataset supporting the conclusions of this article is available in the Figshare repository, [https://doi.org/10.6084/m9.figshare.14559993.v1] [60]

Competing interests: The authors declare that they have no competing interests.

Funding: This work was supported by Czech Science Foundation (grant No. 18-13692S) and Charles University (Research Centre program No. 204056).

Authors' contributions: Not applicable.

Acknowledgements: I would like to thank Anna Pilátová for her useful comments and help with preparing the final version of the article.

\section{References}

1. Tenter AM, Heckeroth AR, Weiss LM. Toxoplasma gondii: from animals to humans. Int J Parasit. 2000;30(1213):1217-58. 
2. Smith NC, Goulart C, Hayward JA, Kupz A, Miller CM, van Dooren GG. Control of human toxoplasmosis. Int J Parasit. 2021;51(2-3):95-121.

3. Luft BJ, Remington JS. Toxoplasmic encephalitis in AIDS. Clin Infect Dis. 1992;15(2):211-22.

4. Garweg JG, Pleyer U. Treatment strategy in human ocular toxoplasmosis: Why antibiotics have failed. J Clin Med 2021, 10(5).

5. Prusa AR, Kasper DC, Sawers L, Walter E, Hayde M, Stillwaggon E. Congenital toxoplasmosis in Austria: Prenatal screening for prevention is cost-saving. PLoS Neglected Tropical Diseases 2017, 11(7).

6. Petersen E, Kijlstra A, Stanford M. Epidemiology of ocular toxoplasmosis. Ocul Immunol Inflamm. 2012;20(2):68-75.

7. Bodaghi B, Touitou V, Fardeau C, Paris L, LeHoang P. Toxoplasmosis: new challenges for an old disease. Eye. 2012;26(2):241-4.

8. Holland GN. LX Edward Jackson memorial lecture - Ocular toxoplasmosis: A global reassessment. Part 1: Epidemiology and course of disease. Am J Ophthalmol. 2003;136(6):973-88.

9. Bertranpetit E, Jombart T, Paradis E, Pena H, Dubey J, Su CL, Mercier A, Devillard S, Ajzenberg D. Phylogeography of Toxoplasma gondii points to a South American origin. Infect Genet Evol. 2017;48:150-5.

10. Grigg ME, Dubey JP, Nussenblatt RB. Ocular toxoplasmosis: Lessons from Brazil. Am J Ophthalmol. 2015;159(6):999-1001.

11. Lidar M, Langevitz P, Shoenfeld Y. The role of infection in inflammatory bowel disease: initiation, exacerbation and protection. Isr Med Assoc J. 2009;11(9):558-63.

12. Severance EG, Alaedini A, Yang SJ, Halling M, Gressitt KL, Stallings CR, Origoni AE, Vaughan C, Khushalani S, Leweke FM, et al. Gastrointestinal inflammation and associated immune activation in schizophrenia. Schizophr Res. 2012;138(1):48-53.

13. Shapira Y, Agmon-Levin N, Selmi C, Petrikova J, Barzilai O, Ram M, Bizzaro N, Valentini G, Matucci-Cerinic M, Anaya $\mathrm{JM}$, et al. Prevalence of anti-Toxoplasma antibodies in patients with autoimmune diseases. J Autoimmun. 2012;39(1-2):112-6.

14. Vittecoq M, Elguero E, Lafferty KD, Roche B, Brodeur J, Gauthier-Clerc M, Misse D, Thomas F. Brain cancer mortality rates increase with Toxoplasma gondii seroprevalence in France. Infect Genet Evol. 2012;12(2):496-8.

15. Thomas F, Lafferty KD, Brodeur J, Elguero E, Gauthier-Clerc M, Misse D. Incidence of adult brain cancers is higher in countries where the protozoan parasite Toxoplasma gondii is common. Biol Let. 2012;8(1):101-3.

16. El-Saadi O, Welham J, Saha S, MaCaulay C, Collingwood L, Chant D, Torrey EF, McGrath J. The incidence and prevalence of schizophrenia: Preliminary results from a systematic review. Schizophr Res. 2002;53(3):32-2.

17. Torrey EF, Bartko JJ, Lun ZR, Yolken RH: Antibodies to Toxoplasma gondii in patients with schizophrenia: A metaanalysis. Schizophrenia Bulletin 2007, 33:729-736.

18. Torrey EF, Bartko JJ, Yolken RH. Toxoplasma gondii and other risk factors for schizophrenia: An update. Schizophr Bull. 2012;38(3):642-7.

19. Flegr J, Horáček J. Toxoplasma-infected subjects report an obsessive-compulsive disorder diagnosis more often and score higher in obsessive-compulsive inventory. Eur Psychiat. 2017;40:82-7.

20. Sutterland AL, Fond G, Kuin A, Koeter MW, Lutter R, van Gool T, Yolken R, Szoke A, Leboyer M, de Haan L. Beyond the association. Toxoplasma gondii in schizophrenia, bipolar disorder, and addiction: systematic review and metaanalysis. Acta Psychiatr Scand. 2015;132(3):161-79.

21. Lindová J, Kuběna AA, Šturcová A, Křivohlavá R, Novotná M, Rubešová A, Havlíček J, Kodym P, Flegr J. Pattern of money allocation in experimental games supports the stress hypothesis of gender differences in Toxoplasma gondiiinduced behavioural changes. Folia Parasitol. 2010;57:136-42. 
22. Lindová J, Novotná M, Havlíček J, Jozífková E, Skallová A, Kolbeková P, Hodný Z, Kodym P, Flegr J. Gender differences in behavioural changes induced by latent toxoplasmosis. Int J Parasit. 2006;36:1485-92.

23. Flegr J, Zitkova S, Kodym P, Frynta D. Induction of changes in human behaviour by the parasitic protozoan Toxoplasma gondii. Parasitology. 1996;113:49-54.

24. Flegr J. Influence of latent Toxoplasma infection on human personality, physiology and morphology: pros and cons of the Toxoplasma-human model in studying the manipulation hypothesis. J Exp Biol. 2013;216(1):127-33.

25. Flegr J, Escudero DQ. Impaired health status and increased incidence of diseases in Toxoplasma-seropositive subjects - an explorative cross-sectional study. Parasitology. 2016;143(14):1974-89.

26. Flegr J, Prandota J, Sovickova M, Israili ZH. Toxoplasmosis - A global threat. Correlation of latent toxoplasmosis with specific disease burden in a set of 88 countries. PLOS One 2014, 9(3).

27. Havlíček J, Gašová Z, Smith AP, Zvára K, Flegr J. Decrease of psychomotor performance in subjects with latent 'asymptomatic' toxoplasmosis. Parasitology. 2001;122:515-20.

28. Neyer LE, Grunig G, Fort M, Remington JS, Rennick D, Hunter CA. Role of interleukin-10 in regulation of T-celldependent and T-cell-independent mechanisms of resistance to Toxoplasma gondii. Infect Immun. 1997;65(5):167582.

29. Kaňková Š, Holáň V, Zajícová A, Kodym P, Flegr J. Modulation of immunity in mice with latent toxoplasmosis - the experimental support for the immunosupression hypothesis of Toxoplasma-induced changes in reproduction of mice and humans. Parasitol Res. 2010;107:1421-7.

30. Buzoni-Gatel D, Dubremetz JF, Werts C. Molecular cross talk between Toxoplasma gondii and the host immune system. M S-Medecine Sciences. 2008;24:191-6.

31. Fenoy IM, Chiurazzi R, Sanchez VR, Argenziano MA, Soto A, Picchio MS, Martin V, Goldman A. Toxoplasma gondii infection induces suppression in a mouse model of allergic airway inflammation. PLoS One 2012, 7(8).

32. Flegr J, Stř́ž I. Potential immunomodulatory effects of latent toxoplasmosis in humans. Bmc Infect Dis. 2011;11:274.

33. Kankova S, Flegr J, Calda P. An elevated blood glucose level and increased incidence of gestational diabetes mellitus in pregnant women with latent toxoplasmosis. Folia Parasitologica 2015, 62.

34. R Core Team. R: A language and environment for statistical computing. R Foundation for Statistical Computing. In. Vienna. Austria; 2018.

35. Kim S. ppcor: An R package for a fast calculation to semi-partial correlation coefficients. Communication for Statistical Applications Methods. 2015;22(6):665-74.

36. Flegr J, Flegr P: Doing exploratory analysis in R with a package Explorer v. 1.0. figshare 2021.

37. Benjamini Y, Hochberg Y. Controlling the false discovery rate: A practical and powerful approach to multiple testing. Journal of the Royal Statistical Society Series B-Methodological. 1995;57(1):289-300.

38. Novotná M, Havlíček J, Smith AP, Kolbeková P, Skallová A, Klose J, Gašová Z, Písačka M, Sechovská M, Flegr J. Toxoplasma and reaction time: Role of toxoplasmosis in the origin, preservation and geographical distribution of Rh blood group polymorphism. Parasitology. 2008;135:1253-61.

39. Flegr J, Novotná M, Fialová A, Kolbeková P, Gašová Z. The influence of RhD phenotype on toxoplasmosis- and ageassociated changes in personality profile of blood donors. Folia Parasitol. 2010;57:143-50.

40. Flegr $\mathrm{J}$, Preiss $\mathrm{M}$, Klose J. Toxoplasmosis-associated difference in intelligence and personality in men depends on their Rhesus blood group but not ABO blood group. PLoS One 2013, 8(4).

41. Sýkorová K, Flegr J. Faster life history strategy manifests itself by lower age at menarche, higher sexual desire, and earlier reproduction in people with worse health. Sci Rep 2021, 11(1).

42. Leroy EM, Gouilh MA, Brugere-Picoux J. The risk of SARS-CoV-2 transmission to pets and other wild and domestic animals strongly mandates a one-health strategy to control the COVID-19 pandemic. One Health 2020, 10. 
43. Newman A, Smith D, Ghai RR, Wallace RM, Torchetti MK, Loiacono C, Murrell LS, Carpenter A, Motoff S, Rooney JA, et al: First reported cases of SARS-CoV-2 infection in companion animals - New York, March-April 2020. Mmwr-Morbidity and Mortality Weekly Report 2020, 69(23):710-713.

44. Hosie MJ, Hofmann-Lehmann R, Hartmann K, Egberink H, Truyen U, Addie DD, Belak S, Boucraut-Baralon C, Frymus T, Lloret A, et al: Anthropogenic infection of cats during the 2020 COVID-19 pandemic. Viruses 2021, 13(2).

45. Drozdz M, Krzyzek P, Dudek B, Makuch S, Janczura A, Paluch E. Current state of knowledge about role of pets in zoonotic transmission of SARS-CoV-2. Viruses-Basel 2021, 13(6).

46. Giraldo-Ramirez S, Rendon-Marin S, Jaimes JA, Martinez-Gutierrez M, Ruiz-Saenz J. SARS-CoV-2 clinical outcome in domestic and wild cats: A systematic review. Animals 2021, 11(7).

47. Dileepan M, Di D, Huang QF, Ahmed S, Heinrich D, Ly H, Liang YY. Seroprevalence of SARS-CoV-2 (COVID-19) exposure in pet cats and dogs in Minnesota, USA. Virulence. 2021;12(1):1597-609.

48. Michelitsch A, Schoen J, Hoffmann D, Beer M, Wernike K. The second wave of SARS-CoV-2 circulation-antibody detection in the domestic cat population in Germany. Viruses-Basel 2021, 13(6).

49. Calvet GA, Pereira SA, Ogrzewalska M, Pauvolid-Correa A, Resende PC, Tassinari WD, Costa AD, Keidel LO, da Rocha ASB, da Silva MFB, et al: Investigation of SARS-CoV-2 infection in dogs and cats of humans diagnosed with COVID-19 in Rio de Janeiro, Brazil. PLoS One 2021, 16(4).

50. Neira V, Brito B, Aguero B, Berrios F, Valdes V, Gutierrez A, Ariyama N, Espinoza P, Retamal P, Holmes EC, et al. A household case evidences shorter shedding of SARS-CoV-2 in naturally infected cats compared to their human owners. Emerg Microbes Infect. 2021;10(1):376-83.

51. Pagani G, Lai A, Bergna A, Rizzo A, Stranieri A, Giordano A, Paltrinieri S, Lelli D, Decaro N, Rusconi S, et al: Human-tocat SARS-CoV-2 transmission: Case report and full-genome sequencing from an infected pet and Its owner in Northern Italy. Pathogens 2021, 10(2).

52. Decaro N, Balboni A, Bertolotti L, Martino PA, Mazzei M, Mira F, Pagnini U. Italian Association Vet I: SARS-CoV-2 infection in dogs and cats: Facts and speculations. Front Vet Sci 2021, 8.

53. Lauzi S, Stranieri A, Giordano A, Lelli D, Elia G, Desario C, Ratti G, Decaro N, Paltrinieri S. Do dogs and cats passively carry SARS-CoV-2 on hair and pads? Viruses-Basel 2021, 13(7).

54. Flegr J, Horáček J. Toxoplasmosis, but not borreliosis, is associated with psychiatric disorders and symptoms. Schizophr Res. 2018;197:603-4.

55. Matowicka-Karna J, Dymicka-Piekarska V, Kemona H. Does Toxoplasma gondii infection affect the levels of IgE and cytokines (IL-5, IL-6, IL-10, IL-12, and TNF-alpha). Clin Dev Immunol. 2009;374696:374696.

56. Flegr J, Horáček J. Negative effects of latent toxoplasmosis on mental health. Front Psychiatry 2020, 10.

57. Flegr J, Kuba R, Kopecký R. Rhesus-minus phenotype as a predictor of sexual desire and behavior, wellbeing, mental health, and fecundity. PLoS One. 2020;15(7):e0236134.

58. Flegr J. Predictors of Toxoplasma gondii infection in Czech and Slovak populations: the possible role of cat-related injuries and risky sexual behavior in the parasite transmission. Epidemiol Infect. 2017;145(7):1351-62.

59. Flegr J, Preiss M. Friends with malefit. The effects of keeping dogs and cats, sustaining animal-related injuries and Toxoplasma infection on health and quality of life. PLoS One 2019, 14(11).

60. Flegr J. The association between covid-19 and toxoplasmosis. Dataset. Figshare, 2021.

\section{Figures}




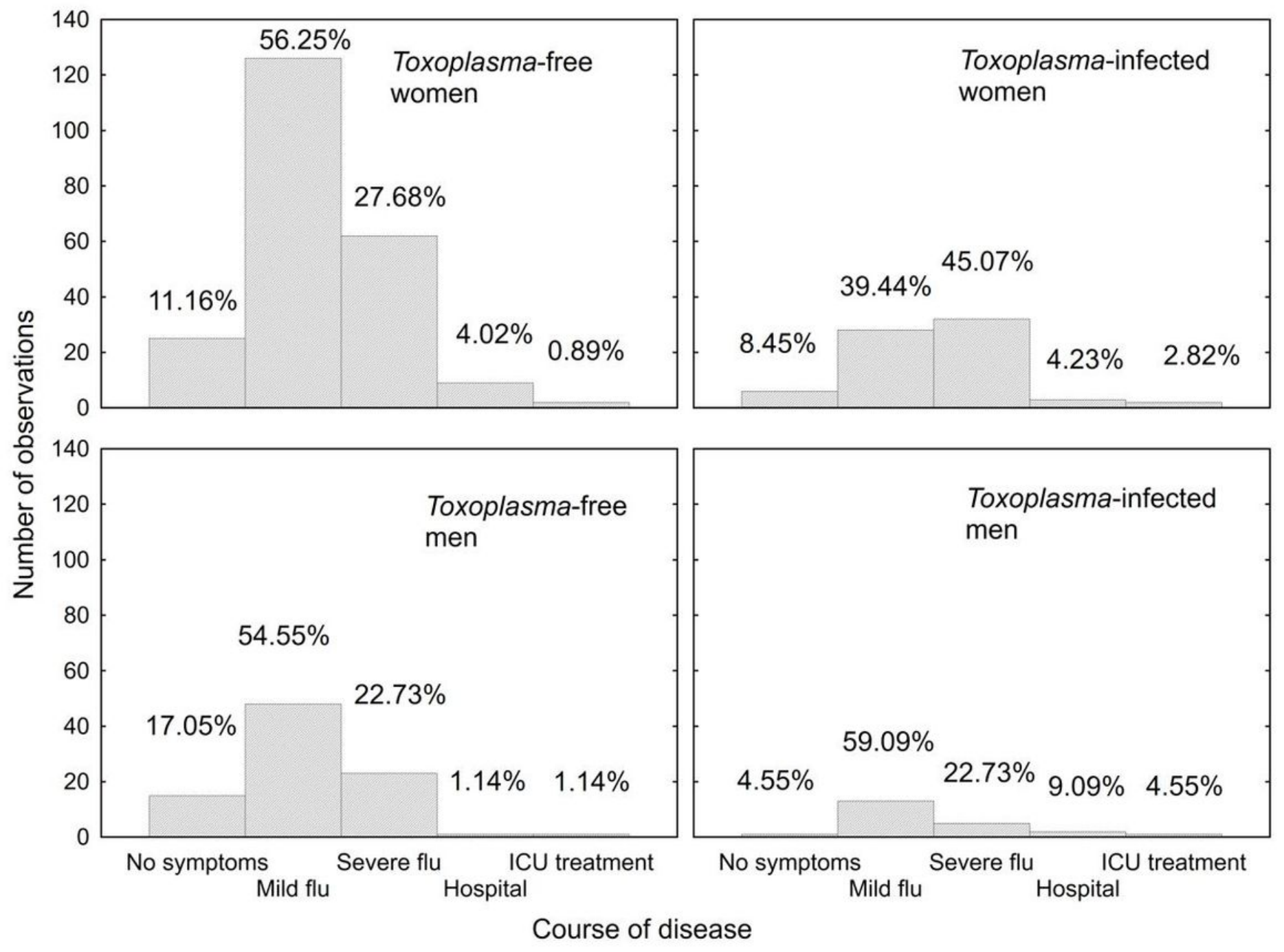

Figure 1

The course of Covid-19 in Toxoplasma-free and Toxoplasma-infected men and women 